\title{
Best Approximants for Bounded Functions and the Lattice Operations
}

\author{
A. O. Chiacchlo, J. B. Prolla and M. S. M. Roversi
}

ABSTRACT. If $V$ is a closed and non-empty subset of $\ell_{\infty}(T)$, the Banach space of all real-valued bounded functions on a set $T$, then existence of best approximants from $V$ and Lipschitz continuity of the metric projection in the Hausdorff metric are proved, whenever $V$ has the following lattice operation property: $((\omega+\varepsilon) \wedge h) \vee(\omega-\varepsilon)$ belongs to $V$, for every $\omega$ and $h$ in $V$ and $\varepsilon>0$.

\section{INTRODUCTION}

Let $T$ be a non-empty set, and let $E=\ell_{\infty}(T)$ be the Banach space of all bounded real-valued functions $f: T \rightarrow \mathfrak{R}$, equipped with the sup-norm

$$
\|f\|=\sup \{|f(t)| ; t \in T\} .
$$

Let $V$ be a closed and non-empty subset of $\ell_{\infty}(T)$. For each $f \in \mathscr{L}_{\infty}(T)$, define

$$
\operatorname{dist}(f ; V)=\inf \{\|f-v\| ; v \in V\} .
$$

A best approximant to f from $V$ is an element $v \in V$ such that

$$
\|f-v\|=\operatorname{dist}(f ; \eta) .
$$

Let $P_{V}(f)$ denote the (possibly empty) set of all such $v$ 's. The set-valued mapping $f \rightarrow P_{V}(f)$ is called the metric projection onto $V$. In this paper we study some properties of $V$ that imply that $P_{V}(f) \neq \emptyset$ and moreover

$$
d_{H}\left(P_{V}(f), P_{V}(g)\right) \leq 2\|f-g\|
$$

1991 Mathematics Subject Classification: 4IA65, 41 A50, 46E05.

Editorial Complutense. Madrid, 1992. 
for all $f$ and $g$ in $E$. Here $d_{H}$ denotes the Hausdorff metric (see definition below). Condition ( ${ }^{*}$ ) implies that $P_{V}$ admits a continuous selection: i.e., there exists a continuous proximity map $\pi: E \rightarrow V$, such that

$$
\pi(n) \in P_{V}(f) \text { for all } f \in E .
$$

Essentially, we prove that any closed non-empty subset $V \subset \ell_{\infty}(T)$ such that $((w+\varepsilon) \wedge h) \vee(w-\varepsilon)$ belongs to $V$, whenever $w, h \in V$, is proximinal, i.e., $P_{Y}\left(D \neq \emptyset\right.$ for every $f \in \mathscr{L}_{\infty}(T)$, and $\left(^{*}\right)$ is valid. In fact, we prove more, namely that relative Chebyshev centers exists, i.e., cent $(B ; V) \neq \emptyset$ for all bounded and non-empty subsets $B \subset \ell_{\infty}(T)$ and moreover

$$
d_{H}(\operatorname{cent}(B ; V), \operatorname{cent}(A ; V)) \leq 2 d_{H}(B ; A)
$$

for every $B$ and $A$ bounded non-empty subsets of $\ell_{\infty}(T)$.

The proof consists in showing that such subsets $V \subset \ell_{\infty}(T)$ enjoy a property here called "property $(C)$ " in the Banach space $\ell_{\infty}(T)$ and that property $(C)$ is responsible for proximinality of $V$ and for $\left({ }^{*}\right)$ and $\left({ }^{* *}\right)$. (See Theorem 1 below).

We then apply this result to various concrete situations, proving new results and extending and generalizing many cases that were known in the literature through the use of deep arguments, or of $a d$ hoc methods.

The connexion between best approximants and lattice operations in $\ell_{\infty}(T)$ was also used by Kripke and Holmes [14], in order to get best approximants by interposition.

This paper is organized as follows: in $\$ 2$ we fix our notation and terminology; in $\$ 3$ we show that property $(C)$ implies existence and continuity of best approximants; in $\$ 4$ we give concrete examples.

\section{NOTATION AND TERMINOLOGY}

For any Banach space $E$, the open and closed balls of center $a$ and radius $r$ are denoted, respectively, by $B(a ; r)$ and $\bar{B}(a ; r)$. If $V$ is any non-empty subset of $E$ and $a \in E$, then

$$
\operatorname{dist}(a ; V):=\inf \{\|a-v\| ; \quad v \in V\} .
$$

We denote by $P_{V}(a)$ the set of all best approximants to $a$ from $V$, i.e.,

$$
P_{y}(a):=\{v \in V ;\|v-a\|=\operatorname{dist}(a ; V)\} .
$$


If $P_{V}(a) \neq \emptyset$ for all $a \in E$, we say that $V$ is proximinal in $E$. If $B \subset E$ is any bounded non-empty subset, then

$$
\operatorname{rad}(B ; V):=\inf \left\{\sup _{f \in B}\|f-v\| ; v \in V\right\}
$$

is called the relative Chebyshev radius of $B$ with respect to $V$. When $V=E$ we write simply $\operatorname{rad}(B)$ and call it the Chebyshev radius of $B$. An element $v \in V$ such that

$$
\sup _{f \in B}\|f-v\|=\operatorname{rad}(B ; V)
$$

is called a relative Chebyshev center of $B$ with respect to $V$, and we denote by cent $(B ; V)$ the set of all such elements. When $V=E$, we write simply cent $(B)$ and its elements are called the Chebyshev centers of $B$.

When cent $(B ; V \neq 0$ for any bounded $B \subset E$, we say that $V$ has the relative Chebyshev center property in $E$. When cent $(B) \neq \emptyset$ for all such $B$, we say that $E$ admits Chebyshev centers.

If $\mathscr{D}(E)$ denotes the class of all bounded and non-empty subsets of $E$, the Hausdorff metric $d_{H}$ is defined to be

$$
d_{H}(A, B):=\inf \{r>0 ; A \subset B+r U, B \subset A+r U\}
$$

for all $A$ and $B$ in $\mathscr{B}(E)$, where $U$ denotes the closed unit ball of $E$, i.e., $U=\{v \in E ;\|v\| \leq 1\}$.

If $T$ is any non-empty set, we denote by $\ell_{\infty}(T)$ the vector space of all bounded real-valued functions defined on $T$. When we endow $\ell_{\infty}(T)$ with the sup-norm

$$
\| f \mid=\sup \{|f(t)| ; \quad t \in T\}
$$

it becomes a Banach space. When $T$ is a topological space, then the vector subspace of all elements of $\mathscr{\ell}_{\infty}(T)$ which are continuous on $T$ is denoted by $C_{b}(T)$. Since it is closed in $\ell_{\infty}(T)$, it is a Banach space too. When $T$ is compact, then all continuous real-valued functions on $T$ are bounded, i.e., $C(T)=C_{b}(T)$. For any topological space $T$, the space $C_{b}(T)$ is isometrically, algebraically and lattice isomorphic to $C(K)$ for some compact Hausdorff space $K$. When $T$ is a completely regular Hausdorff space, then we may take $K$ to be the Stone-Cech compactification of $T$. The set of all $f \in \ell_{\infty}(T)$ such that $f(t) \geq 0$, for any $t \in T$, is denoted by $\ell_{\infty}^{+}(T)$. For any subset $A \subset \ell_{\infty}(T)$, $A^{+}:=A \cap \ell_{\infty}^{+}(T)$. 


\section{EXISTENCE AND CONTINUITY OF BEST APPROXIMANTS}

Definition 1. Let $V$ be a closed non-empty subset of a Banach space $E$, and let $\mathscr{B}$ be a class of bounded non-empty subsets of $E$. We say that the pair $(V, \mathscr{B})$ has property $(C)$ in $E$, if given $B \equiv, w \in V, r>0$ and $0<\varepsilon<1$ such that $V \cap \cap \cap_{f \in B} B(f ; r) \neq 0$ and $\|f-w\|<1+\varepsilon$, for all $f \in B$, there exists $v \in V$ such that $\|v-w\| \leq \varepsilon$ and $\|f-v\| \leq r$ for all $f \in B$.

Let us say that $V$ has property $(C)$ in $E$, if the pair $(V, \mathscr{B}(E))$ has property $(C)$ in $E$, where $\mathscr{B}(E)$ is the class of al' bounded non-empty subsets of $E$. Clearly, if $V$ has property $(C)$ in $E$, and $F$ is a closed vector subspace such that $V \subset F \subset E$, then $V$ has property $(C)$ in the Banach space $F$ too.

If $V$ is a closed vector subspace and the class $\mathscr{B}$ in invariant under translations, then we may take $w=0$ when proving that $(V, \mathscr{B})$ has property $(C)$.

When $\mathscr{B}$ is the class of all singletons of $E$ and $V$ is a closed vector subspace, then $(V, \mathscr{B})$ has property $(C)$ if, and only if, $V$ has the $1 \frac{1}{2}-$ ball property defined by D. T. Yost [23].

Theorem 1. Let $V$ be a closed non-empty subset of a Banach space E. If $V$ has property (C) in $E$, and $F$ is any closed vector subspace of $E$ containing $V$, then

(1) cent $(B ; V) \neq \emptyset$, for every bounded and non-empty subset $B$ of $F$.

(2) The map $B \rightarrow \operatorname{cent}(B ; V)$ is Lipschitz $d_{H}$-continuous, with Lipschitz constant not greater than 2 , i.e.

$$
d_{n}(\operatorname{cent}(K ; V), \operatorname{cent}(L ; V)) \leq 2 d_{I I}(K, L)
$$

for any pair $K, L$ of bounded and non-empty subsets of $F$.

(3) $V$ is proximinal in $F$.

(4) $d_{H}\left(P_{V}(f), P_{V}(g)\right) \leq 2\|f-g\|$ for any pair $f, g$ in $F$.

(5) The metric projection $P_{V}$ admits a continuous selection.

Proof. (3) follows from' (1). On the other hand (2) $\Rightarrow(4) \Rightarrow(5)$. It remains to prove (1) and (2). 
Proof of (1): Let $B$ be a bounded and non-empty subset of $F$. We may assume that $r=\operatorname{rad}(B ; V)>0$. Choose $w_{1} \in V$ with $\left\|w_{1}-f\right\| \leq r+2^{-i}$, for every $f \in B$. Then

$$
V \cap \bigcap_{f \in B} \bar{B}\left(f ; r+2^{-2}\right) \neq \mathfrak{Q} \text { and }\left\|w_{1}-f\right\|<r+2^{-1}+2^{-2}
$$

By property $(C)$, there exists $w_{2} \in V$ such that $\left\|w_{2}-w_{1}\right\| \leq 2^{-1}$ and $\left\|w_{2}-f\right\| \leq r+2^{-2}$ for all $f \in B$. Suppose $w_{1}, w_{2}, \ldots, w_{k} \in V$ have been chosen with

(i) $\left\|w_{n+1}-w_{n}\right\| \leq 2^{-n} \quad(n=1,2,3, \ldots, k-1)$

(ii) $\left\|w_{n}-f\right\| \leq r+2^{-n} \quad(n=1,2,3, \ldots, k)$, for all $f \in B$.

Then $V \cap \cap_{f \in B} \bar{B}\left(f ; r+2^{-(k+1)}\right) \neq \emptyset$ and $\left\|w_{k}-f\right\|<r+2^{-k}+2^{-(k+1)}$ for all $f \in B$. By property $(C)$ there exists $w_{k+1} \in V$ such that $\left\|w_{k+1}-w_{k}\right\| \leq 2^{-k}$ and $\left\|w_{k+1}-f\right\| \leq r+2^{-(k+1)}$, for all $f \in B$. By induction one gets a sequence $\left\{w_{n}\right\}$ satisfying (i) and (ii) for all $n=1,2,3, \ldots$ By (i), the sequence $\left\{w_{n}\right\}$ is Cauchy and by (ii) its limit, say $w$, satisfies $\|w-f\| \leq r=\operatorname{rad}(B ; V)$ for all $f \in B$. Hence $w \in \operatorname{cent}(B ; V)$.

Remark. The proof presented above is, mutatis mutandis, the proof that the $1 \frac{1}{2}$-ball property implies proximinality (see Lemma 1.1, Yost [23]).

The following lemma is going to be used in the proof of part (2) of Theorem 1.

Lemma 1. If $V$ has property $(C)$ in $E$, then, given any bounded and nonempty set $B \subset E$, any $w \in V$ and any $\varepsilon>0$ satisfying $\|f-w\|<\operatorname{rad}(B ; V)+\varepsilon$ for all $f \in B$, there exists $v \in \operatorname{cent}(B ; V)$ such that $\|v-w\| \leq \varepsilon$.

Proof. If $\operatorname{rad}(B ; V)=0$, then $B$ is a singleton, say $\{f$ and $f \in V$. Then cent $(B ; V)=\{f\}$ and $\|f-w\|<\varepsilon$.

If $\operatorname{rad}(B ; V)=r>0$, then cent $\left(E_{;} V\right)=V \cap \cap_{f \in B} \bar{B}(f ; r) \neq 0$. By property (C), there exists $v \in V$ such that $\|v-w\| \leq \varepsilon$ and $\|v-f\| \leq r$ for all $f \in B$. Hence $v \in \operatorname{cent}(B ; V)$.

Proof of (2): Let $\lambda=2 d_{H}(K, L)+\varepsilon$, where $\varepsilon>0$ is arbitrary. Then $d_{H}(K, L)<\lambda / 2$. Hence $K \subset L+\rho U$ and $L \subset K+\rho U$ for some $\rho<\lambda / 2$, where 
$U$ is the closed unit ball of $E$. Choose $v \in \operatorname{cent}(K ; V)$ and $w \in \operatorname{cent}(L ; V)$. Let $g \in L$. Then $g=f+x$, where $f \in K$ and $\|x\| \leq \rho$. Hence

$$
\begin{aligned}
\|v-g\| \leq & \|v-f\|+\|f-g\| \leq \operatorname{rad}(K ; V)+\rho \\
& \leq \sup _{k \in K}\|k-w\|+\rho .
\end{aligned}
$$

On the other hand, for any $k \in K$ we have $k=m+y$, where $m \in L$ and $\|y\| \leq \rho$. Hence

$$
\|k-w\| \leq\|k-m\|+\|m-w\| \leq \rho+\operatorname{rad}(L ; V),
$$

and then $\sup _{k \in K}\|k-w\| \leq \rho+\operatorname{rad}(L ; V)$. Therefore

$$
\|v-g\| \leq \operatorname{rad}(L ; V)+2 \rho<\operatorname{rad}(L ; V)+\lambda
$$

for all $g \in L$. By Lemma 1 there exists $u \in \operatorname{cent}(L ; V)$ such that $\|u-v\| \leq \lambda$. Hence cent $(K ; V) \subset$ cent $(L ; V)+\lambda U$. Similarly, one has cent $(L ; V) \subset$ cent $(K ; V)+\lambda U$. Therefore.

$$
\therefore d_{H}(\operatorname{cent}(K ; V) \text {, cent }(L ; V)) \leq \lambda=2 d_{H}(K ; L)+\varepsilon .
$$

Since $\varepsilon>0$ was arbitrary, $d_{H}(\operatorname{cent}(K ; V), \operatorname{cent}(L ; V)) \leq 2 d_{H}(K ; L)$

Corollary 1. If $V$ has the $1 \frac{1}{2}$-ball property in $E$, then

$$
d_{H}\left(P_{V}(f), P_{V}(g)\right) \leq 2\|f-g\|
$$

for all $f$ and $g$ in $E$. Hence $P_{V}$ admits a continuous selection.

Remark. The Corollary above generalizes the corresponding result of Holmes, Scranton and Ward [10] which says that $\left({ }^{*}\right)$ holds if $V$ is an $M$-ideal in $E$. Recall that $M$-ideals have the $1 \frac{1}{2}$-ball property, and that property $(C)$ reduces to the $1 \frac{1}{2}$-ball property when $\mathscr{B}$ is the class of all singletons of $E$.

\section{EXAMPLES}

In this section we will present many examples of closed non-empty subsets of $\ell_{\infty}(T)$ which have property $(C)$ in $\ell_{\infty}(T)$. The following theorem will provide a wide class of such examples. 
Theorem 2. (1): Let $V$ be a closed and non-empty subset of $L_{\infty}(T)$ such that for each $0<\varepsilon<1$

$$
w, h \in V \Longrightarrow((w+\varepsilon) \wedge h) \vee(w-\varepsilon) \in V .
$$

Then $V$ has property $(C)$ in $\mathscr{C}_{\infty}(T)$.

$0<\varepsilon<1$

(2) Let $V$ be a closed subspace of $\ell_{\infty}(T)$ such that for each

$$
h \in V \Longrightarrow(\varepsilon \wedge h) \vee(-\varepsilon) \in V .
$$

Then $V$ has property $(C)$ in $\ell_{\infty}(T)$.

Proof. (1) Let $B \subset \ell_{\infty}(T)$ be a bounded and non-empty subset, let $w \in V, \quad r>0$ and $0<\varepsilon<1$ be given with $V \cap \cap \cap_{f \in \theta} \bar{B}(f ; r) \neq 0$ and $\|f-w\|<r+\varepsilon$ for all $f \in B$. Choose $h \in V$ such that $\|f-h\| \leq r$, for all $f \in B$. Let $v=((w+\varepsilon) \wedge h) \vee(w-\varepsilon)$. Then $v \in V$ and $\|v-w\| \leq \varepsilon$. We claim that $\|f-v\| \leq r$ for all $f \in B$. Indeed, let $x \in T$ and $f \in B$ be given.

Case 1. $|h(x)-w(x)| \leq \varepsilon$.

Then $v(x)=h(x)$ and $|f(x)-v(x)|=|f(x)-h(x)| \leq r$.

Case 2. $h(x)-w(x)>\varepsilon$.

Then $v(x)=w(x)+\varepsilon$ and $-r \leq f(x)-h(x)<f(x)-w(x)-\varepsilon<r+\varepsilon-\varepsilon=r$.

Case 3. $h(x)-w(x)<-\varepsilon$.

Then $v(x)=w(x)-\varepsilon$ and $-r=-(r+\varepsilon)+\varepsilon<f(x)-w(x)+\varepsilon<f(x)$ $-h(x) \leq r$.

Hence our claim is true, and $V$ has property $(C)$ in $\ell_{\infty}(T)$.

(2) This follows from (1) and the remark that, when $V$ is a closed subspace then we may take $w=0$ when proving that $(V, \mathscr{B}(E))$ has property $(C)$ in $E=\mathscr{L}_{\infty}(T)$. The fact that $\mathscr{B}(E)$, the class of all bounded non-empty subsets of $E$, is invariant under tanslations is also needed.

Remark 1. It is perhaps worth noticing that we do not assume in Theorem 2 that $V$ itself is a lattice. In Example 8 we give an example of a 
vector subspace $V$ which is not a lattice, but such that (ii) is true and so $V$ has property $(C)$ in $\ell_{\infty}(T)$.

Let us note the following Corollary of Theorem 2.

Corollary 2. Let $V$ be a closed and non-empty subset of $L_{\infty}(T)$ verifying the hypothesis of Theorem 2. Then $V$ is proximinal in $\ell_{\infty}(T \times S)$ for every non-empty set $S$. If $T$ is a topological space and $V$ is a closed and non-empty subset of $C_{b}(T)$ verifying the hypothesis of Theorem 2, then $V$ is proximinal in $l_{\infty}(T \times S)$, for every non-empty set $S$, and, a fortiori, in $C_{b}(T \times S)$, for every non-empty topological space $S$.

Proof. By Theorem 1 , cent $(B ; V) \neq 0$ for every bounded subset $B \subset C_{\infty}(T)$. Now given $f \in \ell_{\infty}(T \times S)$, define the section $f_{s}$ as the mapping $t \rightarrow f(t, s)$. Clearly, $f_{s} \in \ell_{\infty}(T)$ and $B=\left\{f_{s} ; s \in S\right\}$ is bounded in $\ell_{\infty}(T)$. It is easy to see that any element of cent $(B ; V)$ provides a best approximant to $f$ in $V$ considered as a subset of $\ell_{\infty}(T \times S)$.

Remark 2. Let $V_{1}$ and $V_{2}$ be two closed non-empty subsets of $\mathscr{L}_{\infty}(T)$ such that both $V_{1}$ and $V_{2}$ satisfy the conditions stated in part (1) of Theorem 2. Then, whenever $V_{1} \cap V_{2}$ is non-empty, $V_{1} \cap V_{2}$ also satisfies the conditions stated in part (1) of Theorem 2. Hence $V_{1} \cap V_{2} \neq \emptyset$ implies that $V_{1} \cap V_{2}$ has property $(C)$ in $\ell_{\infty}(T)$. A similar remark applies to part (2) of Theorem 2. Now, given a non-empty subset $N$ of $T$, the closed subset

$$
V(N)=\left\{f \in \ell_{\infty}(T) ; f(t)=0 \text { for all } t \in N\right\} \quad \because \cdot
$$

is non-empty and satisfies the conditions stated in both (1) and (2) of Theorem 2 . Hence, whenever $V$ is a subset of $\ell_{\infty}(T)$ satisfying the conditions in (1) or (2) of Theorem 2, then $V_{0}=V \cap V(N)$, when it is non-empty, also has property $(C)$ in $\ell_{\infty}(T)$. Therefore, to each example $V$ given in the sequel, there is a corresponding example provided $V_{0}=V \cap V(N)$ is non-empty. We shall abstain of stating in each instance that such a corresponding example exists.

Notice also that, when $T$ is a non-empty topological space, then $C_{b}(T)$ satisfies the conditions of part (1) and (2) of Theorem 2. Hence, whenever $V \subset \ell_{\infty}(T)$ satisfies the conditions of part (1) or part (2) of Theorem 2 , then $V \cap C_{b}(T)$ also satisfies the same conditions when it is non-empty, and therefore provides a further example of a set satisfying property $(C)$ in $\dot{\ell}_{\infty}(T)$.

When $T$ is a locally compact non-empty topological space, a similar remark applies to $V \cap C_{0}(T)$, where $C_{0}(T)$ denotes the space of all continuous real-valued functions defined on $T$ and vanishing at infinity. 
Remark 3. Suppose $S$ and $T$ are non-empty sets (resp. topological spaces), and let $\varphi: S \rightarrow T$ be a map of $S$ onto $T$ (resp. a continuous map of $S$ onto $T$ ). The map $\varphi^{*}: \mathscr{L}_{\infty}(T) \rightarrow \ell_{\infty}(S)$ defined by $\varphi^{*}(f)=f \circ \varphi$ defines a linear isometry of $\mathscr{L}_{\infty}(T)$ onto a closed subset of $\mathscr{L}_{\infty}(S)$. Since $\varphi^{*}$ is linear and preserves products and lattice operations, if $V \subset \mathscr{L}_{\infty}(T)$ is a closed subspace, or a closed subalgebra or a closed lattice, $\varphi^{*}(V) \subset \ell_{\infty}(S)$ has the same properties in $\ell_{\infty}(S)$. When $\varphi$ is continuous and $V \subset C_{b}(T)$, then $\varphi^{*}(V) \subset C_{b}(S)$. Since $\varphi^{*}$ preserves linear and lattice operations, we see that whenever $V \subset$ $\ell_{\infty}(T)$ satisfies the hypothesis of Theorem 2, the same is true of $\varphi^{*}(V)$ as a subset of $\ell_{\infty}(S)$. Hence $\varphi^{*}(V)$ satisfies (1) $-(5)$ of Theorem I. In particular, $\varphi^{*}(V)$ is proximinal in $\ell_{\infty}(S)$. Note that, by the properties of the map $\varphi^{*}$ what one can conclude in general is only that, if $V$ is a proximinal subset of $\ell_{\infty}(T)$, then $\varphi^{*}(V)$ is proximinal in $\varphi^{*}\left(\ell_{\infty}(T)\right)$. But we see that when $V$ satisfies the hypothesis of Theorem $2, \varphi^{*}(V)$ is proximinal in the whole space $\ell_{\infty}(S)$.

As already noted in Remark $2, C_{b}(T)$ satisfies the hypothesis of Theorem 2. Hence $\varphi^{*}\left(C_{b}(T)\right)$ satisfies (1) - (5) of Theorem I. In particular, $\varphi^{*}\left(C_{b}(T)\right)$ is proximinal in $C_{b}(S)$, a result due to Mazur, for compact $S$ and $T$. The subspaces of $C_{b}(S)$ of the form $\varphi^{*}\left(C_{b}(T)\right)$ are called Stone-Weierstrass subspaces. By the Stone-Weierstrass Theorem they are precisely the closed unital subalgebras of $C_{b}(S)$, when $S$ is compact.

Remark 4. K. S. Lau [15, Proposition 4.4] showed that when $V$ satisfies the hypothesis of part (1) of Theorem 2, then $V$ is $U$-proximinal, with $\varepsilon(\rho)=\rho$, in his terminology, and that $\left(^{*}\right)$ of Corollary 1 above holds for such $V$.

Let us now give a list of examples of subsets $V \subset \mathscr{L}_{\infty}(T)$ to which Theorem 2 can be applied. Hence for all of them (1) - (5) of Theorem 1 are valid. Some of these conclusions were known in the literature, others are new and some are generalizations or extensions of known results. Whenever the older results were known to us we indicate the references.

Example 1. Let $T$ be a non--empty topological space. As already remarked $V=C_{b}(T)$ satisfies the hypothesis of part (1) of Theorem 2 and therefore has property $(C)$ in $\ell_{\infty}(T)$. The proximinality of $C_{b}(T)$ in $\ell_{\infty}(T)$, for the particular case in which $T$ is a paracompact space was proved by Holmes and Kripke in 1965. (See [9], and also Holmes [7, pg. 125].) The fact that $P_{V}$ (for $V=C_{b}(T)$ ) is Lipschitz $d_{H}$-continuous with Lipschitz constant not greater than 2 is due to Kripke (see Holmes [7, pg. 173]) again in the particular case when the space $T$ is paracompact. Now observe that (4) of Theorem 1 is true for any $f$ and $g$ in any closed vector subspace $F$ such that $C_{b}(T) \subset F \subset \mathscr{\ell}_{\infty}(T)$. As an example take $F$ to be the space of all bounded Borel real-valued functions on $T$. Note that $C_{b}(T)$ has the $1 \frac{1}{2}$-ball property in $F$, 
but Holmes [7] points out that $C_{b}(T)$ lacks the 2-ball property in $F$. The result that $C(T)$ admits Chebyshev centers is due to Kadets and Zamyatin [12] for $T=[a, b]$, and to Garkavi [6] for a compact Hausdorff space $T$. Their results were extended by Mach [16]: indeed, it follows from Theorems 3 and 4 of [16] that, for any topological space $T$, the space $C_{b}(T)$ has the relative Chebyshev center property in $\ell_{\infty}(T)$ and the map $B \rightarrow \operatorname{cent}\left(B ; C_{b}(T)\right)$ is lower semicontinuous. The result that $C_{b}(T)$ admits Chebyshev centers, for any topological space $T$, was also noticed by Franchetti and Cheney [5].

Example 2. Let $a$ and $b$ be two elements in $\ell_{\infty}(T)$, and let $V=[a, b]$, i.e.,

$$
V=\left\{f \in \ell_{\infty}(T) ; a(x) \leq f(x) \leq b(x), \quad \text { for all } \quad x \in T\right\}
$$

Then $V$ satisfies the conditions stated in part (1) of Theorem 2. The proximinality of $[a, b]$ in $\ell_{\infty}(T)$ is implicit in Franchetti and Cheney [5, Lemma 3.5]: any order interval in a Banach lattice is proximinal. Roversi [20, Proposition 2.6] proved that $[a, b]$ has the relative Chebyshev center property in $\ell_{\infty}(T)$ (that is part (1) of Theorem 1). Notice that when $a<b$ are two real numbers, and if we identify $a$ and $b$ with the respective constant functions that they define, then

$$
V=\left\{f \in \ell_{\infty}(T) ; \quad f(T) \subset[a, b]\right\}
$$

where now $[a, b]$ denotes an interval in $\mathfrak{R}$.

Another example that falls in this category is the following. Let $u \in \ell_{\infty}(T)$ and $\varphi \in \ell_{\infty}^{+}(T)$ be given. Let

$$
V=\left\{f \in \ell_{\infty}(T) ; \quad|f-u| \leq \varphi\right\} .
$$

Note that $|f-u| \leq \varphi$ means $|f(x)-u(x)| \leq \varphi(x)$ for all $x \in T$. This is equivalent to $a \leq f \leq b$, where $a=u-\varphi$ and $b=u+\varphi$. Hence $V=[u-\varphi$, $u+\varphi$ ]. In particular, we get that any closed ball in $\ell_{\infty}(T)$ (resp. in $C_{b}(T)$, if $T$ is a topological space) is proximinal in $\ell_{\infty}(T)$.

Suppose now that $T$ is a topological space, and consider $V=[a, b] \cap C_{b}(T)$, with $a$ and $b$ given in $\ell_{\infty}(T)$ with $a \leq b$. By Remark 2 , we know that $V$ has property $(C)$ in $\ell_{\infty}(T)$, if it is non-empty. It is well-known that this is the case when $T$ is a normal space, $a$ is upper semicontinuous and $b$ is lower semicontinuous: The proximinality of $V=[a, b] \cap C_{b}(T)$ in this case was proved by Franchetti and Cheney [5, Theorem 3.3]. Further cases in which $V \neq \emptyset$ are listed in Roversi [20, Theorem 3.13]. 
Example 3. Let $V$ be a closed non-empty sublattice of $\ell_{\infty}(T)$ such that $w+r$ belongs to $V$, whenever $w \in V$ and $r \in \mathfrak{R}$. By part (1) of Theorem 2, $V$ has property $(C)$ in $\ell_{\infty}(T)$. Roversi [20, Proposition 2.4] had proved that $V$ has the relative Chebyshev center property in $C_{\infty}(T)$.

An example is provided by the set of functions with a prescribed modulus of continuity. Let $(T, d)$ be a metric space, and let $\omega: \mathfrak{R}_{+} \rightarrow \mathfrak{R}_{+}$be a nondecreasing function. Let $V=C_{\omega}(T)$ be the class of all $f \in C_{b}(T)$ such that $\omega_{f}(\delta) \leq \omega(\delta)$, for all $\delta>0$, where

$$
\omega_{f}(\delta)=\sup \{|f(t)-f(s)| ; \quad d(t, s) \leq \delta\} .
$$

A special case is given by the subclass $\operatorname{Lip}_{\alpha}(M)$ of all $f \in \operatorname{Lip}_{\alpha}$ with Lipschitz constant not greater than $M$ :

$$
V=\left\{f \in C_{\infty}(T) ;|f(t)-f(s)| \leq M d(t, s)^{\alpha}\right\} .
$$

One can generalize this in the following manner. Let $T$ be a non-empty set, and let $\Delta$ be a non-empty subset of $T \times T$. For each function $\varphi: \Delta \rightarrow[0$, $\infty$ ), define

$$
\begin{aligned}
& V=\left\{f \in \ell_{\infty}(T) ;|f(t)-f(s)| \leq \varphi(s, t) \text { for all }(s, t) \in \Delta\right\}, \\
& V_{i}=\left\{f \in \ell_{\infty}(T) ; f(s) \leq f(t)+\varphi(s, t) \quad \text { for all } \quad(s, t) \in \Delta\right\},
\end{aligned}
$$

and

$$
V_{d}=\left\{f \in \ell_{\infty}(T) ; f(s) \geq f(t)-\varphi(s, t) \text { for all }(s, t) \in \Delta\right\}
$$

Clearly, $V=V_{i} \cap V_{d \cdot}$. A special case is given by a pre-ordered set $(T, \leq)$ and $\Delta=\{(s, t) \in T \times T ; s \leq t\}$. When $\varphi \equiv 0, V_{i}$ (resp. $\left.V_{d}\right)$ is the set of all nondecreasing (resp. non-increasing) bounded functions. The case in which $\varphi$ depends only on $s$ was studied by Smarzewski [21]. Further examples of closed lattices $V$ such that $\omega+r$ belongs to $V$, for all $r \in \mathfrak{R}$ and $\omega \in V$, are given by the sets of all bounded functions on a topological space that are lower semicontinuous, respectively upper semicontinuous.

As another example, let $\left\{A_{\alpha} ; \alpha \in I\right\}$ be a family of finite subsets of some non-empty set $T$, say

$$
A_{\alpha}=\left\{t_{1}^{\alpha}, t_{2}^{\alpha}, \ldots, t_{n(\alpha)}^{\alpha}\right\} .
$$

Let $V$ be the subset of $\ell_{\infty}(T)$ of all $f \in \ell_{\infty}(T)$ satisfying

$$
f\left(t_{1}^{\alpha}\right) \geq f\left(t_{2}^{\alpha}\right) \geq \ldots \geq f\left(t_{n(\alpha)}^{\alpha}\right)
$$


for each $\alpha \in I$. Then $V$ is a closed sublattice of $\ell_{\infty}(T)$ such that $f+r$ belongs to $V$, whenever $f \in V$ and $r \in \mathfrak{R}$.

When $T=T_{1} \times T_{2}$, where $T_{1}$ and $T_{2}$ are pre-ordered sets, then new examples are given by the sets of all functions in $\ell_{\infty}(T)$ that are nondecreasing in each variable, or non-increasing in each variable, or that are non-decreasing in one variable and non-increasing in the other variable.

Example 4. Let $V$ be a closed sublattice of $\mathscr{L}_{\infty}(T)$ containing the constants and such that $V+V \subset V$. Then $V$ satisfies the hypothesis of Example 3. For instance, take $V$ to be a closed sublattice of $\mathscr{C}_{\infty}(T)$ containing the constants; which is also a convex cone. This shows that Theorems. 1 and 2 generalize an Approximation Theorem of Blatter and Seever [2], [3]. For such a $V$ they proved that

$$
V_{0}=\{f \in V ; f(t)=0 \text { for all } t \in N\},
$$

where $N$ is a (possibly empty) subset of $T$, is proximinal in $\ell_{\infty}(T)$. Their proof uses the theory of interposition of functions that they developed. In [3] they establish a formula for dist $(f, V)$ in terms of the quasi-proximity defined by $V$ on $T$. The approximation theorem of Blatter and Seever extends an approximation theorem of Nachbin [17, Appendix, \$5, Theorem 6] which proves that any closed lattice convex cone $V \subset C(T)$, containing the constants, is proximinal in $C(T)$, for $T$ a compact Hausdorff space. (When $N=\emptyset$, then Blatter and Seever's result follows from Nachbin's). Nachbin also proved a formula for dist $(f ; V)$.

Example 5. Let $T$ be a compact Hausdorf space. A non-empty subset $A \subset C(T)$ is called a semi-algebra (Bonsall [4]) if $A A \subset A, A+A \subset A$ and $\lambda A \subset A$ for each $\lambda \geq 0$. The semi-algebra $A$ is called a semi-algebra with identity if it contains the positjve constants. Given a non-negative integer $n \geq 0$, a semi-algebra $A$ is said to be of type $n$ if $f^{n} /(1+f)$ belongs to $A$, whenever $f \in A$. Every semi-algebra of type 0 is a semi-algebra with identity, and if $A$ is a semi-algebra of type $n \geq 0$, then $A \subset C^{+}(T)$. Notice that if $A$ is of type $n$, then it is of type $n+1$.

Let now $A$ be a closed semi-algebra of type 1 and with identity. Define a pre-order $\leq$ on $T$ by declaring $s \leq t$ whenever $f(s) \leq f(t)$ for all $f \in A$. By Theorem 7 of Bonsall [4], an element $g \in C^{+}(T)$ belongs to $A$ if, and only if, $g(s) \leq g(t)$ whenever $s \leq t$. Hence it is straightforward to verify that $((\omega+\varepsilon)$ $\wedge h) \vee(\omega-\varepsilon)$ belongs to $A$ whenever $\omega$ and $h$ belong to $A$.

If $A \subset C(T)$ is a subalgebra, then $A^{+}=\{f \in A ; f \geq 0\}$ is a semi-algebra, and its closure $V=\overline{A^{+}}$is a closed semi-algebra. If $A$ contains the constants, 
then $V$ is a semi-algebra of type 0 . Hence $V$ is a closed semi-algebra of type I with identity. If $A \subset C(T)$ is a subalgebra which does not contain the nonzero constants (this is equivalent, by the Stone-Weierstrass Theorem, to say that $N=\{x \in T ; f(x)=0$ for all $f \in A\} \neq 0)$, then $V=\overline{A^{+}}$is still a closed semialgebra, but we cannot apply the previous argument. Instead, one can reason as follows. Let $w, h \in V$ and $\varepsilon>0$ be given. Define

$$
f=((w+\varepsilon) \wedge h) \vee(w-\varepsilon) .
$$

If $f(x) \neq f(y)$, then $w(x) \neq w(y)$ or $h(x) \neq h(y)$. Since $w, h \in \overline{A^{+}}$, there is some $g \in A^{+}$such that $g(x) \neq g(y)$. On the other hand, if $f(x)>0$, then $w(x)>0$ or $h(x)>0$. Since $w, h \in \overline{A^{+}}$, there is some $g \in A^{+}$such that $g(x)>0$. By Corollary 4, Prolla [18], the function $f$ belongs to $V$. Notice that this argument applies regardless of the fact that $A$ contains or not the identity.

Example 6. Let $A$ be a subalgebra of $\ell_{\infty}(T)$. Let $T_{d}$ be the set $T$ equipped with the discrete topology. Then $\ell_{\infty}(T)=C_{b}\left(T_{d}\right)$ and so $\ell_{\infty}(T)$ is isometrically, algebraically and lattice isomorphic to $C(K)$, where $K$ is the Stone-Cech compactification of $T_{d}$. Hence we may suppose that $A$ is a subalgebra of $C(K), K$ a compact Hausdorff space. Let $V=\bar{A}$. Let $h \in V$ and $\varepsilon>0$ be given. Define $f=(\varepsilon \wedge h) \vee(-\varepsilon)$. Suppose $f(x) \neq f(y)$. Then $h(x) \neq h(y)$. Since $h \in \bar{A}$, there is some $g \in A$ such that $g(x) \neq g(y)$. Suppose, on the other hand that, $f(x) \neq 0$. Then $h(x) \neq 0$. Since $h \in \bar{A}$, there is some $g \in A$ such that $g(x) \neq 0$. By the Stone-Weierstrass Theorem, $f \in \vec{A}$. By part (2) of Theorem 2, $V=\bar{A}$ has property $(C)$ in $C(K)$

If $T$ is any topological space and $A \subset C_{b}(T)$, then we consider $A$ as a subalgebra of $\ell_{\infty}(T)$, and apply the previous result to get that $\bar{A}$ has property $(C)$ in $\ell_{\infty}(T)$ and consequently $(1)-(5)$ of Theorem 1 are true when $E=\mathscr{C}_{\infty}(T)$ and $F$ is any closed vector subspace of $\ell_{\infty}(T)$ containing $A$. In particular, we may take $F=C_{b}(T)$ or more generally any $F$ such that $A \subset F \subset C_{b}(T)$.

If $T$ is locally compact, the same results hold for a subalgebra $A$ of $C_{0}(T)$. Just notice that $F=C_{0}(T)$ is a closed subspace of $C_{b}(T)$. Hence the closure of $A$ in $C_{0}(T)$ has property $(C)$ in $\ell_{\infty}(T)$.

Some of the conclusions (1) - (5) of Theorem 1 in the case of a closed subalgebra $A \subset C_{b}(T)$ are well-known, but were proved using less elementary methods than the one presented here. For example, Smith and Ward [22] proved, using interposition theorems, that any closed subalgebra $A$ of $C(T)$, for compact $T$, has the relative Chebyshev center property in $C(T)$.

Example 7. Let $T$ be a compact topological space, and let $D(T)$ be the subset of $C(T)$ of all continuous functions $f: T \rightarrow \Re$ such that $0 \leq f(t) \leq 1$, for 
all $t \in T$. Let $A \subset D(T)$ be a closed non-empty subset with property $V$ and containing one constant $0<c<1$. Property $V$ is defined as follows: $1-\varphi$ and $\varphi \psi$ belong to $A$, whenever $\varphi$ and $\psi$ belong to $A$. Then $A$ has property $(C)$ in $\ell_{\infty}(T)$. Indeed, let $h \in A$ and $w \in A$ be given. Let $f=((w+\varepsilon) \wedge h) \vee(w-\varepsilon)$, where $0<\varepsilon<1$. If $f(x) \neq f(y)$, then either $w(x) \neq w(y)$ or $h(x) \neq h(y)$. By Corollary 1, Prolla [19], $f \in \bar{A}=A$. By Theorem 2, $A$ has property (C) in $\ell_{\infty}(T)$. Notice that, even though it is known that $A$ is a lattice (Jewett [12], Theorem 1), this fact alone is not sufficient to prove that $f \in A$, because $w+\varepsilon$ or $w-\varepsilon$ may not belong to $A$.

Example 8. Let $M$ be a non-empty collection of maps of a non-empty set $T$ into itself. Let $\rho=1$ or $\rho=-1$. Let

$$
V=\left\{f \in \ell_{\infty}(T) ; f(g(t))=\rho f(t), \quad \text { for each } \quad g \in M \quad \text { and } t \in T\right\} .
$$

When $\rho=1, V$ is a closed linear lattice containing all the constants and Theorem 2, part (1) applies. When $\rho=-1, V$ is a closed subspace of $\ell_{\infty}(T)$, and the only constant belonging to $V$ is zero. We claim that condition (ii) of Theorem 2 is verified. Indeed, let $h \in V$ and $\varepsilon>0$ be given. For any $g \in M$ we have, for $f=(\varepsilon \wedge h) \vee(-\varepsilon)$ and $x \in T$ :

$$
\begin{aligned}
f(g(x)) & =(\varepsilon \wedge h(g(x))) \vee(-\varepsilon) \\
& =(\varepsilon \wedge(-h(x))) \vee(-\varepsilon) \\
& =(-((-\varepsilon) \vee h(x)))) \vee(-\varepsilon) \\
& =-(((-\varepsilon) \vee h(x)) \wedge \varepsilon \\
& =-f(x) .
\end{aligned}
$$

Hence $f \in V$, and Theorem 2, part (2) applies.

Notice that $V$ may fail to be a lattice, in the case $\rho=-1$. For example, take $T=[-1,1]$, and let $M$ consist of the single function $g(x)=-x$. When $\rho=1$ we get the even functions and $\rho=-1$ we get the odd functions. In the case $\rho=-1, V$ is not a lattice, as $f(x)=x$ shows: $f \wedge 0$ and $f \vee 0$ do not belong to $V$. So $V$ is not even a lower or an upper semi-lattice.

Example 9. Let $T$ be a non-empty locally compact topological space. For $f \in C_{b}(T)$ we say that

$$
\lim _{t \rightarrow \infty} f(t)=v
$$

if, given $\varepsilon>0$ there exists a compact subset $K \subset T$ such that $|f(t)-v|<\varepsilon$ for all $t \in T, t \notin K$. Following Amir and Deutsch [1], $\mathrm{C}_{\Re}(T)$ denotes the closed 
vector subspace of $C_{b}(T)$ of all functions that have limit at infinity. It is easy to see that $V=C_{\mathfrak{R}}(T)$ verifies the hypothesis of Theorem 2, part (2). If $T=\mathfrak{n}$, then $V=c$, the closed vector subspace of $l_{\infty}$ of all sequences such that $\lim _{n \rightarrow \infty} x_{n}$ exists. Note that

$$
C_{0}(T)=\left\{f \in C_{R}(T) ; \lim _{t \rightarrow \infty} f(t)=0\right\}
$$

also satisfies the hypothesis of Theorem 2, part (2). When $T=\mathfrak{M}, V=c_{0}$. Hence for both $V=c$ and $V=c_{0}$, and for $E=\ell_{\infty}$ all conclusions (1) $-(5)$ of Theorem 1 are true. In the special case of approximation in $\ell_{\infty}$ by the subspace $c_{0}$, Amir and Deutsch [1, Proposition 4.1] proved that in part (4) of Theorem 1 , the constant 2 is hest possible.

Example 10. Let $\varphi: \ell_{\infty}(T) \rightarrow \mathfrak{h}$ be a linear lattice homomorphism. Then $\varphi$ is a monotonic linear functional, hence $\varphi$ is order-bounded. By Corollary 3.5.6 of Jameson [11], $\varphi$ is continuous. Let $V$ be its kernel. Then $V$ is a closed linear subspace. Notice that $V$ is solid, i.e., $f \in V$ and $|g| \leq|f|$ imply $g \in V$. Indeed,

$$
0 \leq|\varphi(g)|=\varphi(|g|) \leq \varphi(|f|)=|\varphi(f)|=0
$$

Hence $\varphi(g)=0$, and $g \in V$. Now, for every solid subset $V \subset \ell_{\infty}(T)$ one has $h \in V \Rightarrow(\varepsilon \wedge h) \vee(-\varepsilon) \in V$. Indeed, let $f=(\varepsilon \wedge h) \vee(-\varepsilon)$. Then $|f| \leq|h|$, and therefore $f \in V$. By Theorem 2, part (2), the closed subspace $V$ has property (C) in $\ell_{\infty}(T)$. For example, if $s \in T$, then $\delta_{s}(f)=f(s)$ is a linear lattice homomorphism. Now if $\alpha$ is a monotonic linear functional such that $0 \leq \varphi \leq \delta_{s}$, then $\varphi$ is a linear lattice homomorphism.

\section{References}

[1] D. AMIR and F. DEUTSCH, Approximation by cerlain subspaces in the Banach space of continuous vector-valued functions. J. Approximation Theory 27 (1979), 254-270.

[2] J. BLATTER and G. L. SEEVER, Inclusions, interposition and approximation. In: Approximation Theory (G. G. Lorentz, editor), Academic Press, New York, 1973, 257-261.

[3] J. BLATTER and G. L. SEEVER, Interpolation and latice cones of functions. Trans. Amer. Math. Soc. 222 (1976), 65-96.

[4] F. F. BonsalL, Semi-algebras of continuous functions. Ptoc. London Math. Soc. (3) $10(1960), 122-140$.

[5] C. FRANChetTl and E. W. ChENEY, Simullaneous approximation and restricted Chebyshev centers in function spaces. In: Approximation Theory and Applications (Z. Ziegler, editor), Academic Press, New York, 1981, 65-88. 
[6] A. L. GARKAVI, The best possible net and the best possible.cross-section of a set in normed space. Amer. Math. Soc. Transl. 39 (1964), 111-132.

[7] R. B. Holmes, A Course on Optimization and Best Approximation, Lecture Notes in Mathematics, Vol. 257. Springer-Verlag, 1972.

[8] R. B. HOLMES, M-Ideals in approximation theory. In: Approximation Theory II (G. G. Lorentz, editor), Academic Press, 1976, 391-396.

[9] R. B. HOLMES and B. KRIPKE, Approximation of bounded functions by continuous functions. Bull. Amer. Math. Soc. 71 (1965), 896-897.

[10] R. B. HOLMES, B. SCRANTON and J. D. WARD, Approximation from the space of compact operators and order M-ideals. Duke Math. J. 42 (1975). 259-269.

[11] G. J. O. JAMESON, Ordered Linear Spaces, Lecture Notes in Mathematics, Vol.

$\therefore$ 141 Springer-Verlag, 1970.'

[12] , R. I. JEWETT, $A$ variation on the Stone-Weierstrass theorem. Proc. Amer, Math. Soc. 14 (1963), 690-693.

[13] I. M. Kadets and V. ZamYatin, Chebyshev centers in the space $C[a, b]$, Theor. Funkcii. Funkcional. Anal. i Priložen 7 (1968), 20-26 (Russian).

[14] : B. KRIPKE and R. HOLMES, Interposition and approximation. Pacific J. Math. 24 (1968), 103-110.

[i5]" 'K. S. LAU, On a sufficient condition for proximity. Trans. Amer. Math. Soc. 251 (1979), 343-356.

[16] J. MACH, Best simultaneous approximation of bounded functions with values in certain Banach spaces. Math. Ann. 240 (1979), 157-164.

[17] L. NACHBIN, Topology and Order. Van Nostrand Math. Studies 4, Van Nostrand, Princeton, 1965.

[18] J. B. ProllA, Approximation by positive elements of subalgebras of real valued functions. In: Topics in Multivariate Approximation (C. K. Chui, L. L. Schumaker and F. I. Utreras, editors), Academic Press, Inc., 1987, 185-192.

[19]' J.'B. PRoLLA, Uniform approximation of continuous functions with values in [0,'1]. In: Procédings International Workshop on Multivariate Approximation and Interpolation (W. Haussmann, K. Jetter, eds.), International Series of

.. Numerical Mathematics; vol: 94, Birkhäuser Verlag, Basel, 1990, pp. 241-247.

[20] M. S. M. RoverSI, Best approximation of bounded functions by continuous functions. J. Approximation Theory 41 (1984), 135-148.

[21] R. SMARzEWSKI, Determination of Chebyshev approximations by nonlinear admissible subsets. J. Approximation Theory 37 (1983), 69-88.

[22] P. W. SMITH and J. D. WARD, Restricted centers in subalgebras of $C(X)$. J. Approximation Theory 15 (1975), 54-59.

[23] - D. T. YosT, Best approximation and intersections of balls in Banach spaces.

Bull. Austral. Math. Soc. 20 (1979), 285-300.

Universidade Estadual de Campinas

IMECC, Caixa Postal 6065

1308 I Campinas, S.P.. Brazil 